\title{
Reduced interhemispheric functional connectivity in the motor cortex during rest in limb-onset amyotrophic lateral sclerosis
}

\author{
Laura M. Jelsone-Swain ${ }^{1}$, Brett W. Fling ${ }^{2}$, Rachael D. Seidler ${ }^{2,3}$, Rebecca Hovatter $^{1}$, Kirsten Gruis $^{4}$ and \\ Robert C. Welsh ${ }^{1,5 *}$
}

Department of Radiology, Basic Radiological Sciences, University of Michigan, Ann Arbor, MI, USA

2 School of Kinesiology, University of Michigan, Ann Arbor, MI, USA

${ }^{3}$ Department of Psychology, University of Michigan, Ann Arbor, MI, USA

${ }^{4}$ Department of Neurology, University of Michigan, Ann Arbor, MI, USA

${ }^{5}$ Department of Psychiatry, University of Michigan, Ann Arbor, MI, USA

\section{Edited by:}

Silvina G. Horovitz, National Institutes of Health, USA

\section{Reviewed by:}

Rasmus Birn, University of Wisconsin, USA

Brian D. Berman, University of

Colorado at Denver Health Sciences Center, USA

*Correspondence:

Robert C. Welsh,

Departments of Radiology and

Psychiatry, 3208B Med Sci 1 5667,

1336 Catherine Street,

University of Michigan, Ann Arbor,

MI 48109, USA.

e-mail:rcwelsh@med.umich.edu
Amyotrophic lateral sclerosis (ALS) is a progressive neurodegenerative disorder of motor neurons that leads to paralysis and eventually death. There is evidence that atrophy occurs in the primary motor cortex (M1), but it is unclear how the disease affects the intrinsic connectivity of this structure. Thus, the goal of this study was to examine interhemispheric coupling of low frequency blood-oxygen-level dependent (BOLD) signal fluctuations in M1 using functional connectivity magnetic resonance imaging during rest. Because disease progression is rapid, high-functioning patients were recruited to assess neural changes in the relatively early stages of ALS. Twenty patients with limb-onset ALS participated in this study. A parceling technique was employed to segment both precentral gyri into multiple regions of interest (ROI), thus increasing sensitivity to detect changes that exist along discretely localized regions of the motor cortex. We report an overall systemic decrease in functional connectivity between right and left motor cortices in patients with limb-onset ALS. Additionally, we observed a pronounced disconnection between dorsal ROI pairs in the ALS group compared to the healthy control group. Furthermore, measures of limb functioning correlated with the connectivity data from dorsal ROI pairs in the ALS group, suggesting a symptomatic relationship with interhemispheric M1 connectivity.

Keywords: ALS, fcMRI, resting-state, primary motor cortex

\section{INTRODUCTION}

Amyotrophic lateral sclerosis (ALS) is the most common adult motor neuron disease with a lifetime risk of approximately 1 in 2000 (Bruijn et al., 2004). Yet, the etiology of 90-95\% of all ALS cases remains unknown. It is reported in the literature an $80 \%$ mortality rate within 5 years of symptom onset (del Aguila et al., 2003). One of the most notable features of ALS is its rapid development of motor impairments. ALS progresses from initial muscle weakness to complete loss of muscle function resulting in death from respiratory failure. Disease progression is associated with dysfunction of both upper and lower motor neurons; however, it is unclear as to which are more directly affected (van der Graaff et al., 2009). Seeley et al. (2009) have shown a direct and potentially unique correlation between disease and affected brain networks, and thus suggest that disease state can be characterized by quantitative descriptors of network metrics such as functional connectivity.

Resting-state functional connectivity magnetic resonance imaging (fcMRI) is a useful method for assessing neural-network connectivity (Biswal et al., 1995). It has been widely used to examine clinical populations, and is currently a method of interest in determining disease-specific neural biomarkers (Grady et al., 2001; Lowe et al., 2002; Koshino et al., 2005; Cao et al., 2006; Cherkassky et al., 2006; Tian et al., 2006; Waites et al., 2006; Wang et al., 2006a,b; Greicius et al., 2007; He et al., 2007; Welsh et al., 2008; Monk et al.,
2009). Additionally, resting-state fcMRI eliminates the interpretation of task effort or demand, which can pose limitations on taskbased fMRI studies. This is partly because resting-state fcMRI does not require patients to engage in any specific task (such as motor movements), which may confound BOLD signal interpretation as a result of task difficulty effects.

Ten prominent resting-state networks have been previously identified (Damoiseaux et al., 2006). Mohammadi et al. (2009) examined five of these, including the default mode, sensorimotor, parietal-temporo-frontal, posterior, and ventral networks, using independent component analysis (ICA) in patients with ALS. Of relevance to the present study, sensorimotor network differences were observed, showing decreased functional connectivity in the premotor cortex. These authors (Mohammadi et al., 2009) examined whole-brain voxel-wise effects. In the current study a seed-based analysis was used to examine more subtle and systemic changes that might occur in the network involving M1 connections.

The primary goal of the current study was to investigate coupling of interhemispheric low frequency BOLD signal fluctuations in the primary motor cortices (M1) using fcMRI during rest in patients with ALS. This structure within the sensorimotor network was of interest for several reasons. First, it is well established that interhemispheric connections between M1 exist. Even during rest it has been demonstrated that the left and right motor 
cortices exhibit temporally correlated BOLD signal fluctuations (Biswal et al., 1997). Second, M1 is a large and somatotopically organized structure (Penfield and Boldrey, 1937). By examining the entire M1 we might be more sensitive to localize changes related to symptom presentation. Last, seminal work (Lawyer and Netsky, 1953; Smith, 1960; Brownell et al., 1970) described postmortem precentral gyrus nerve fiber degeneration in ALS, which suggests this deterioration occurs during the natural disease process. Therefore we hypothesized that a systemic decrease in functional connectivity across hemispheres would be observed. Specifically, we expected that the precentral gyri would show overall less correlated interhemispheric time-series fluctuations in the ALS group compared to controls.

In the present study, a parceling technique was employed that segmented the primary motor cortices into multiple regions of interest (ROIs). This was done to better delineate changes that might exist along the entire motor strip, thus increasing analysis sensitivity to discretely localized regions of this cortex. This investigation focused on the analysis of limb-only onset ALS patients to control for any potential differences in disease progression between limb and bulbar onset patients. Limb-onset ALS is most common, with the affected limb becoming progressively weaker as the disorder spreads to nearby myotomes. Finally, to identify whether systemic changes occur in M1 prior to moderate-severe symptom presentation, high-functioning and/or early disease stage patients were recruited.

\section{MATERIALS AND METHODS PARTICIPANTS AND BEHAVIORAL DATA}

All ALS participants were recruited through the Department of Neurology, were diagnosed by a neuromuscular physician using the El Escorial criteria, and were followed in the Motor Neuron Disease Clinic at the University of Michigan. Healthy controls were recruited from the surrounding area through community advertisements (flyers and web-pages). All participants gave written consent to participate and the Institutional Review Board (IRB) at the University of Michigan approved this study. Twenty patients with limb-onset ALS (13 males, mean age 58.3 years) within 24 months of symptom onset, and 20 sex-matched healthy volunteers ( 13 males, mean age 57.5 years) were recruited to participate in this study. Participants between groups were matched as best as possible to age.

Several measurements of behavioral and cognitive functioning were taken prior to each participant's scanning session. Physical ability of ALS patients was assessed using the ALS functional rating scale, revised version (ALSFRS-r; Cedarbaum and Stambler, 1997). The ALSFRS-r is a validated rating instrument of ALS patients' functional abilities and has been demonstrated to correlate with physiological measures of the disease (Cedarbaum and Stambler, 1997). The ALSFRS-r instrument is comprised of 12 questions, each measuring the level of impairment for different behaviors, such as handwriting and walking. Each question is scored between four and zero points based on ability level, with a maximum total score of 48 points. The ALS cognitive behavioral screen (ALS-CBS; Woolley-Levine, 2006) was administered to assess general cognitive functioning. Because the CBS was not incorporated until after project initiation, 10 ALS patients and no healthy volunteers were tested. Hand dominance was determined by the Edinburgh Inventory (Oldfield, 1971), and hand strength was measured with a hand-grip dynamometer. The mean of three trials with the dynamometer per right and left hand were calculated to determine each participant's hand strength. These measures are presented in Tables $\mathbf{1}$ and $\mathbf{2}$.

A goal of our study was to examine neural changes in highfunctioning patients that may take place in the early disease stage of ALS. Currently there are no standardized criteria to define disease stage. Therefore we defined our high-functioning ALS subjects as early in his or her disease course such that patients were within 24 months of symptom onset at the time of their scan. In addition, only patients who were ambulatory, without hemiplegia, were able to write, had a negative psychiatric evaluation, did not have dysphasia or breathing problems and were not on a ventilator, and were without other complicating mental disorders or disease were only included in the study.

\section{IMAGE ACOUISITION}

All scanning was performed at the University of Michigan's Functional MRI Laboratory on a GE 3T Excite 2 (General Electric, Milwaukee, WI, USA). During each participant's session, medium-resolution spin-echo, and high-resolution spoiled-gradient recall (SPGR) anatomic images $\left(\mathrm{T}_{1}\right.$-Overlay and $\mathrm{T}_{1}$-SPGR respectively) were collected in the axial plane. $\mathrm{T}_{2}^{*}$ time-series data were acquired in the axial plane (aligned to the anterior-posterior commissure) using a reverse-spiral k-space readout. A total of $180 \mathrm{~T}_{2}^{*}$ -weighted volumes were collected for each participant during each scanning session (repetition time TR $=2 \mathrm{~s}$, 40-slice volumes at $3 \mathrm{~mm}$ slice thickness and no skip, echo-time TE $=30 \mathrm{~ms}, 64 \times 64$ matrix, field-of-view, FOV $=220 \mathrm{~mm}$ ). Four $\mathrm{T}_{2}^{*}$ volumes at the beginning of each time-series acquisition were excited but not

Table 1 | Group average (mean) demographic information for ALS patients and healthy participants. Range for age, hand strength, ALSFRS-r and CBS scores, and months since onset are also presented. Handedness is based on the Edinburgh Inventory (Oldfield, 1971).

\begin{tabular}{lllllllll}
\hline Subject & Age (years) & Gender & $\begin{array}{l}\text { Dominant } \\
\text { hand }\end{array}$ & $\begin{array}{l}\text { Dominant } \\
\text { H.S.(kg) }\end{array}$ & $\begin{array}{l}\text { Non-dominant } \\
\text { H.S.(kg) }\end{array}$ & ALSFRS-r & CBS & M.S.O. \\
\hline ALS $(n=20)$, & $58.35(46-67)$ & $n=13 \mathrm{M}, 7 \mathrm{~F}$ & $n=15 \mathrm{R}, 5 \mathrm{~L}$ & $20.60(0-50.75)$ & $11.94(0-42.42)$ & $39.6(29-46)$ & $13.1(7-19)$ & $17.25(8-24)$ \\
$\mathrm{HC}(n=20)$, & $57.51(47-64)$ & $n=13 \mathrm{M}, 7 \mathrm{~F}$ & $n=19 \mathrm{R}, 1 \mathrm{~L}$ & $32.99(11.21-62.12)$ & $30.62(8.33-54.84)$ & NA & NA & NA \\
\hline
\end{tabular}

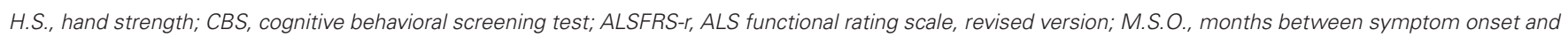
testing date. 
Table 2 | Individual ALS patient demographic information.

\begin{tabular}{|c|c|c|c|c|c|c|c|c|c|c|}
\hline $\begin{array}{l}\text { ALS } \\
\text { subject }\end{array}$ & $\begin{array}{l}\text { Age } \\
\text { (months) }\end{array}$ & Gender & $\begin{array}{l}\text { Dominant } \\
\text { hand }\end{array}$ & $\begin{array}{l}\text { Onset } \\
\text { location }\end{array}$ & $\begin{array}{l}\text { H.S.D. } \\
\text { (kg) }\end{array}$ & ALSFRS-r & CBS & M.S.O. & $\begin{array}{l}\text { ALSFRS- } \\
r \# 4\end{array}$ & $\begin{array}{l}\text { ALSFRS- } \\
\text { r \#8 }\end{array}$ \\
\hline 1 & 708 & M & $\mathrm{R}$ & Hands & 19.33 & 43 & NA & 08 & 3 & 3 \\
\hline 3 & 709 & $\mathrm{~F}$ & $\mathrm{R}$ & $\begin{array}{l}\text { Left upper } \\
\text { extremity }\end{array}$ & 25.16 & 39 & NA & 22 & 3 & 3 \\
\hline 5 & 721 & $\mathrm{~F}$ & $L^{*}$ & $\begin{array}{l}\text { Right upper } \\
\text { extremity }\end{array}$ & 19.00 & 44 & NA & 24 & 3 & 4 \\
\hline 6 & 553 & M & $\mathrm{R}$ & Right leg & 12.67 & 39 & NA & 21 & 3 & 2 \\
\hline 7 & 780 & $\mathrm{~F}$ & $\mathrm{R}$ & Hands & 0.06 & 29 & NA & 12 & 2 & 2 \\
\hline 8 & 721 & M & $\mathrm{R}$ & Left foot & 14.39 & 46 & NA & 23 & 4 & 3 \\
\hline 13 & 732 & $\mathrm{~F}$ & $\mathrm{R}$ & All four limbs & 10.06 & 42 & 9 & 12 & 4 & 3 \\
\hline 14 & 661 & M & $\mathrm{R}$ & Right hand & 9.09 & 43 & 19 & 14 & 3 & 4 \\
\hline 15 & 780 & $\mathrm{~F}$ & $\mathrm{R}$ & Left leg & 7.42 & 33 & 15 & 18 & 3 & 2 \\
\hline 16 & 804 & M & $\mathrm{R}$ & Legs & 3.00 & 40 & 7 & 11 & 4 & 2 \\
\hline 17 & 624 & M & $\mathrm{R}$ & $\begin{array}{l}\text { Left upper } \\
\text { extremity }\end{array}$ & 12.87 & 31 & 13 & 12 & 3 & 2 \\
\hline 18 & 778 & $M$ & $\mathrm{R}$ & Right leg & 0.00 & 45 & 15 & 23 & 4 & 2 \\
\hline 19 & 699 & $M$ & $\mathrm{R}$ & Right Hand & 10.06 & 46 & 17 & 23 & 4 & 4 \\
\hline 20 & 765 & M & $L^{*}$ & $\begin{array}{l}\text { Upper } \\
\text { extremities }\end{array}$ & 0.00 & 33 & 12 & 21 & 3 & 4 \\
\hline
\end{tabular}

H.S.D., hand strength disparity; CBS, cognitive behavioral screening test; ALSFRS-r, ALS functional rating scale, revised version; M.S. O., months between symptom onset and testing date; ALSFRS-r \#8, question number 8 on the questionnaire, which pertains to ambulation; ALSFRS-r \#4, question number 4 on the questionnaire, which pertains to handwriting. Maximum score for these questions is 4 and minimum is 0 . *Patients 2, 5, and 20 reported right hand dominance before symptom

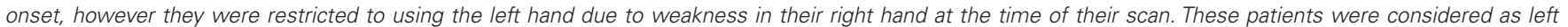
handed based on results from the Edinburgh Inventory (Oldfield, 1971).

recorded in order to achieve thermal equilibrium of magnetization. In addition, physiological recording took place during the scanning session (cardiac and respiratory cycles) using a MRI vendor supplied pulse oximeter and respiratory belt. These data were collected in order to correct for cardiac and respiratory influence on the resting-state signal. Medium-resolution anatomic images ( $\mathrm{T}_{1}$-Overlay) were acquired in the same-slice locations as the $\mathrm{T}_{2}^{*}$ volumes, however at a higher in-plane resolution $(256 \times 256$ matrix, $220 \mathrm{~mm}$ FOV). The $\mathrm{T}_{1}$-SPGR high-resolution images were collected with a $256 \times 256$ matrix, $220 \mathrm{~mm}$ FOV, and with $1.2 \mathrm{~mm}$ slice thickness.

\section{RESTING-STATE TASK}

Resting-state activity was collected over a period of $8 \mathrm{~min}$ for each scanning session. During this time, participants were instructed to view a white crosshair fixed on a black background. This image was projected onto a screen at the head of the scanner bore and viewed with a back-projected mirror, placed on the head coil. Participants were asked to keep their eyes open and to not think about anything in particular to elicit resting-state metabolism (Fox et al., 2005).

\section{fcMRI PREPROCESSING}

In order to reduce noise and artifact, several preprocessing steps were conducted. Using a custom code written in MATAB (Mathworks, Natick, MA, USA; Noll et al., 1991), raw fMRI data were reconstructed offline. Physiological correction of time-series data was implemented in the image domain (Hu et al., 1995; Pfeuffer et al., 2002). This was done because cardiac cycle and respiration give rise to correlated spatial and temporal variance during task execution in fMRI experiments, hence contributing to residual noise and overall decreased statistical power. Removal of physiological confounds is especially important during restingstate acquisition since no overt task is being executed, otherwise presenting the complication of masking true functional connectivity signals or falsely giving rise to functional networks (Lund, 2001; Peltier et al., 2003).

Using FSL's MCFLIRT and SLICETIMER within the fMRI analysis package ${ }^{1}$, motion and slice timing were corrected for, respectively. Realigned images were used for the connectivity analysis, and movement was checked to assure that no more than $0.4 \mathrm{~mm}$

${ }^{1}$ http://www.fmrib.ox.ac.uk/fsl/slicetier/index.html 
translational and $0.1^{\circ}$ rotational movement occurred, thereby minimizing motion-induced spatial-temporal correlations. Using Statistical Parametric Mapping, version 2 (SPM2; Wellcome Trust Center for Neuroimaging ${ }^{2}$ ), each participant's $\mathrm{T}_{1}$-Overlay volume was co-registered to the time-series data; the $\mathrm{T}_{1}$-SPGR was then co-registered to the co-registered $\mathrm{T}_{1}$-overlay image. Spatial normalization to the Montreal Neurological Institute (MNI 152) template of the resulting co-registered $\mathrm{T}_{1}$-SPGR image was then performed using SPM2. The resulting normalization matrix was applied to the slice-time-corrected, physiologically corrected time-series data. These normalized $\mathrm{T}_{2}^{*}$ time-series data were subsequently spatially smoothed with a 5-mm Gaussian kernel. The resulting $\mathrm{T}_{2}^{*}$ images had $3 \mathrm{~mm}$ isotropic voxels.

\section{REGIONS OF INTEREST}

The primary goal of this study was to examine relationships between the primary motor cortices in ALS and to identify the spectrum of changes that may be taking place. Normalized $\mathrm{T}_{1}$ high-resolution images were used to manually create precentral gyrus gray matter masks in both hemispheres for each participant (using the mean location of the hand knob area as an anatomical anchor). A group anatomical ROI mask was constructed for each hemisphere from voxels shared by $\geq 10$ participants. The ROI group masks were parceled along both motor cortices into 41 and 40 individual $6 \mathrm{~mm}$ ROI cubes in the left and right hemisphere, thus allowing for connectivity analyses between localized anatomical regions. The motor cortex is a large structure that is somatotopically organized (Penfield and Boldrey, 1937). Therefore by segmenting this strip into small ROIs we have increased our sensitivity to delineating functional connectivity changes along this cortex, rather than obscuring what is known about the detailed somatotopic organization of M1 (by using large ROIs, for example; Craddock et al., 2010).

The ventral and dorsal-most ROI cube locations correspond to MNI coordinates $(x, y, z$ in $\mathrm{mm})-60.8,-5.7,26.0:-13.6,-29.3,74.1$ and 65.3, 0.6, 19.7: 5.4,-29.3, 78.6 in the left and right hemispheres, respectively. Because precentral gyrus masks were created from shared voxels in the participant group, coordinate locations of left and right ventral/dorsal-most ROI cubes are not perfectly symmetrical. The average precentral gyri masks are shown in Figure 1.

\section{M1 ROI COUPLING CORRELATION ANALYSIS}

The $\mathrm{T}_{2}^{*}$ time-series were detrended to remove slow drift. Additionally, the global volume intensity was regressed from the time-series. To remove any residual physiological nuisance, the global white matter and cerebral spinal fluid (CSF) temporal signals were sequentially regressed from the $\mathrm{T}_{2}^{*}$ time-series. (Fox et al., 2009). Additionally, motion parameters were treated as nuisance and removed via regression. Mean time-series data were extracted from these ROIs and correlated with each cube in the opposite hemisphere, resulting in an $r$-coefficient correlation-gram for each individual. In order to examine global group differences, the $r$-coefficient correlation-grams by group were then compared using the two sample non-parametric Kolmogorov-Smirnov (K-S) goodness-of-fit test (Chakravarti et al., 1967). Because we expected a systemic change

${ }^{2}$ http://www.fil.ion.ucl.ac.uk/spm

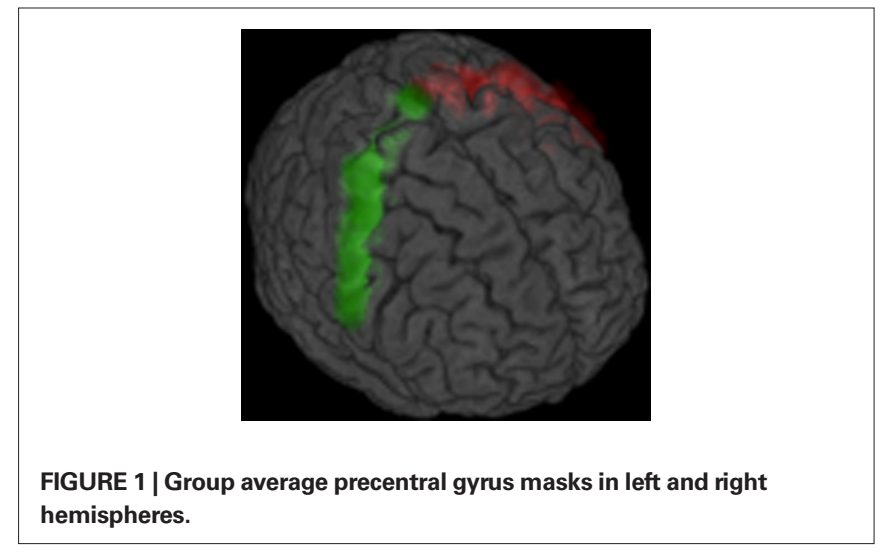

in the distribution of connectivity between the ALS and healthy control groups, the K-S test was well suited to evaluate our hypothesis. Correlation-grams were further evaluated by comparing only near-homologous and homologous-interhemispheric ROIs to assure that differences observed between groups were not due to off-diagonal correlations. In other words, ROI pair correlations that may correspond to non-homologous body regions, such as the foot and face, were removed from this second analysis. A mask was created to only include these homologous- and near-homologous ROIs, which is represented in Figure 2A. Two additional correlation-gram masks were created, which divided the homologous/near-homologous ROIs into ventral (Figure 2B) and dorsal regions (Figure 2C), in order to systemically examine somatotopic subdivisions. Specifically, the dorsal motor cortex is functionally localized to control limb and trunk regions, whereas the ventral cortex corresponds to face regions (Penfield and Boldrey, 1937). Correlation-grams per group were averaged and are presented in Figures 3 and 4. Coordinates along the axes of this map represent ROIs in left and right hemispheres, moving from ventral to dorsal locations.

\section{BEHAVIORAL ANALYSIS}

To examine the possible brain-behavior relationship with limb functioning in the ALS group, behavioral test data were compared with resting-state functional connectivity. Linear regression tests between imaging and limb functioning data were separated into three different analyses based on the average functional connectivity correlation coefficients from (A) diagonal-only; (B) ventral-ventral; (C) and dorsal-dorsal ROI pairs (refer to Figure 2). Functional connectivity measures from these pairs were entered into each regression analysis as the dependent variable. In the ALSFRS-r, questions 4 and 8 pertain to upper and lower limb functioning. These questions assess the ability to write and walk, respectively. Therefore participant scores for these questions were entered into the regression analysis as independent variables. Total scores from the ALSFRS-r were not used because abilities other than limb functioning are measured in this questionnaire, such as breathing. Additionally, hand strength disparity was considered a factor of limb functioning and was entered as a third independent variable into the analysis. Hand strength disparity was calculated as the absolute value of strength difference between the right and left hands. 

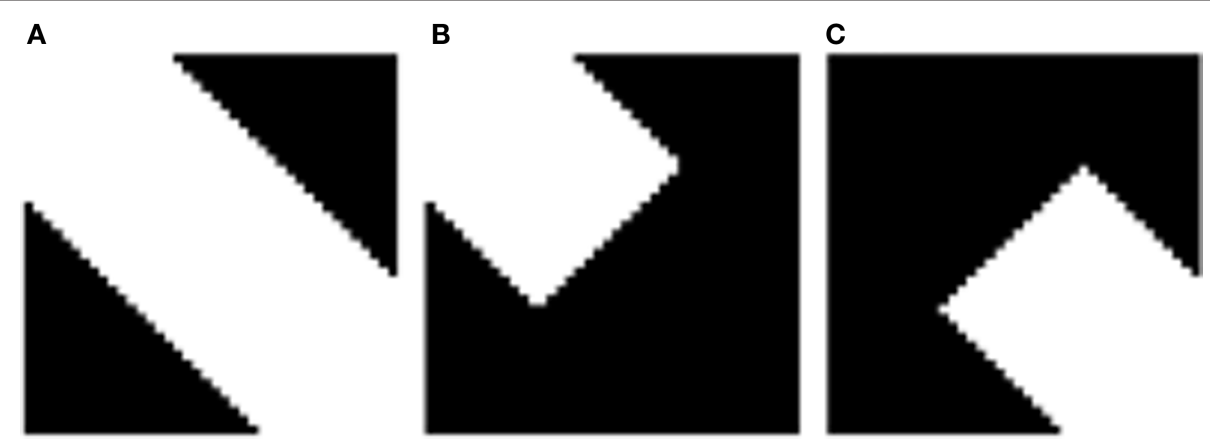

FIGURE 2 | Masks applied to correlation-grams after initial analysis of all ROI pairs. (A) diagonal-only mask; (B) ventral mask; (C) dorsal mask

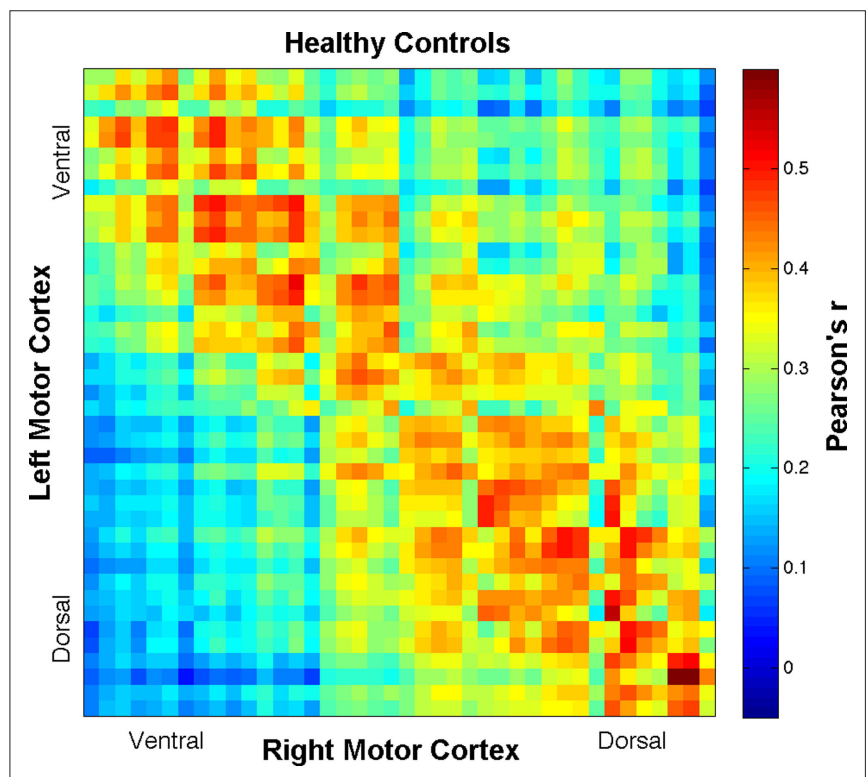

FIGURE 3 | Correlation-gram displaying all ROI Pearson's $r$-correlation coefficient means for the healthy control group. Coordinates along the axes of this map represent ROIs in left and right hemispheres, moving from ventral to dorsal locations.

Bivariate correlation analyses were also conducted, using Pearson's correlation coefficient (two-tailed). In the healthy control group, hand strength disparity was correlated with (A) diagonalonly; (B) ventral-ventral; (C) and dorsal-dorsal ROI pairs. Hand strength disparity, months since onset, ALSFRS-r and CBS scores from the ALS group were entered into a separate correlation analysis. All behavioral analyses were conducted in SPSS, v.17.

\section{RESULTS}

\section{GROUP PHYSICAL AND COGNITIVE BEHAVIORAL DATA}

Group averages for demographic data and behavioral measures are shown in Table 1, and individual patient data are shown in Table 2. According to the ALSFRS-r, the ALS participants as a group were relatively high functioning ( mean $=39.23, \mathrm{SD}=5.06$ ). All patients were ambulatory, as shown by ALSFRS-r question \#8 ( mean $=2.95$, $\mathrm{SD}=0.88)$. In addition, ALSFRS-r question \#4 showed that all but one of the patients could use their hand to write (mean $=3.1$,

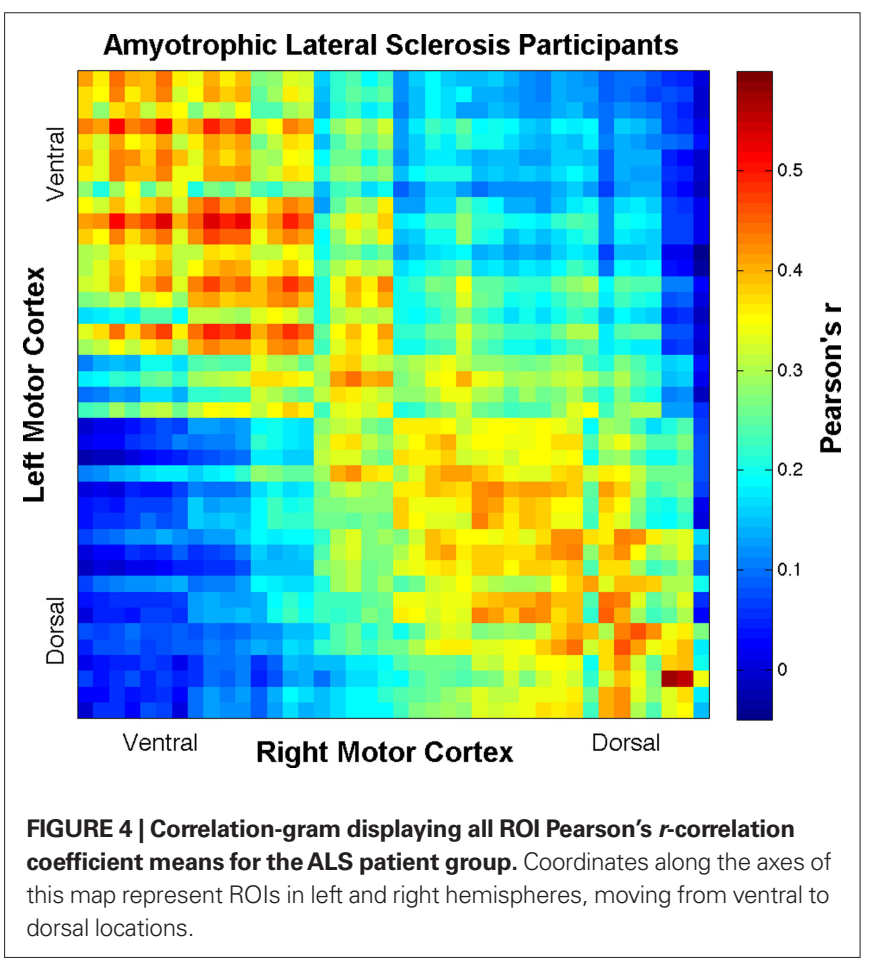

$\mathrm{SD}=0.72$ ). Only one patient reported that they were unable to write, which was due to weakness in their dominant right hand and the resulting ineffective adaptation of writing with the left hand.

\section{RESTING-STATE FUNCTIONAL CONNECTIVITY}

Group comparisons of interhemispheric motor cortex BOLD signal $r$-coefficients, including all ROI pairs, resulted in highly significant K-S differences $(D)$ between ALS and healthy participants $(D=0.19$, $p<7 \times 10^{-26}$ ) with ALS being less connected overall than healthy controls (see Figures 3 and 4). To control for more homologous correspondence, the $\mathrm{K}-\mathrm{S}$ test was performed along the diagonal of the correlation-grams such that mainly homologous regions were included while those regions between which no functional connectivity was expected were excluded. That is, by only including the homologous- and near-homologous regions, we removed any potential for observed statistical difference being driven by negative correlations (Fox et al., 2009). The K-S difference was still found 
to be statistically significant $\left(D=0.14, p<9 \times 10^{-9}\right)$ and not driven solely by the off-diagonal components of the correlation-grams. Further, separating the gyri into ventral (first 20 ROI cubes) and dorsal (remaining ROI cubes) regions, a large effect was observed in the dorsal half $\left(D=0.23, p<3 \times 10^{-12}\right)$, corresponding to limb and trunk locations of the motor homunculus. The ventral half still showed a significant decrease in connectivity, but not to the same extent $(D=0.11, p<0.005)$.

Participant movement while scanning could potentially present confounded functional connectivity results, especially if one group moved more than the other. We tested this possible confound by running independent post hoc $t$-tests between groups on the standard deviations of the motion parameters. These results were null, $p>0.05$, indicating no differences in movement between the ALS and healthy control group.

\section{BEHAVIORAL CORRELATION RESULTS}

The relationship between limb functioning and dorsal motor cortex connectivity in the ALS group was further examined in a regression analysis, with ALSFRS-r questions 4 and 8 and hand strength disparity as predictors of dorsal-dorsal M1 connectivity. This regression analysis was significant, $F(3,19)=3.35, p=0.045$ $(R=0.62)$. In this model, hand strength disparity significantly predicted dorsal ROI connectivity, $t(19)=-2.22, p=0.04$, and question 4 reached near significance, $t(19)=1.81, p=0.08$. Hand strength disparity and question 4 also significantly correlated with dorsal-dorsal M1 connectivity, $r=-0.51, p=0.01$ and $r=0.44, p=0.03$, respectively. Question 8 was insignificant in this model, $t(19)=0.44, p=0.67 ; r=-0.13, p=0.29$. Both remaining regression analyses conducted examining diagonal-diagonal and ventral-ventral M1 connectivity in the ALS patients yielded null results. The healthy control group did not show a significant correlation with hand strength disparity and functional connectivity for any of the three analyses: (A) diagonal-diagonal; (B) ventral-ventral; and (C) dorsal-dorsal pairs. Figure 5 depicts the relationship between dorsal ROI connectivity and hand strength disparity for both ALS and healthy control groups.

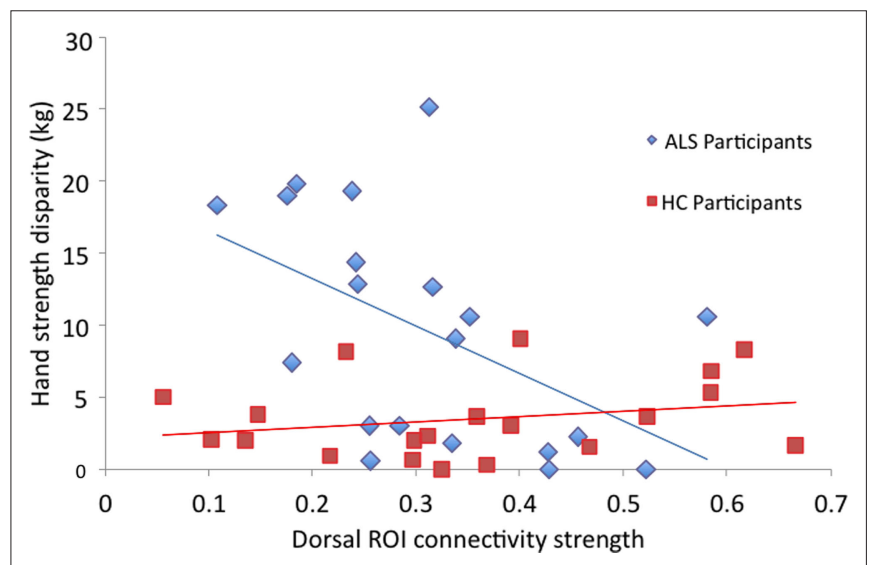

FIGURE 5 | Scatter-plot showing the correlation between average dorsal ROI interhemispheric connectivity per individual and hand strength disparity, across groups. Only results from the ALS group are significantly (inversely) correlated.
Hand strength disparity did not significantly correlate with months since onset, ALSFRS-r or CBS scores in the ALS group. Additionally, ALSFRS-r and CBS scores did not correlate. There was a trend toward significance between months since onset and ALSFRS-r scores $(r=0.41, p=0.07)$ and an even stronger trend between months since onset and CBS scores $(r=0.62$, $p=0.058)$.

\section{DISCUSSION GENERAL DISCUSSION}

The current study provides new evidence for M1 network impairment in ALS, importantly showing for the first time an interhemispheric disconnect. We observed a decrease in overall functional connectivity between parceled motor cortices in the ALS group, as shown by less correlated resting-state low frequency BOLD signal fluctuations across hemispheres. Importantly, this decrease was seen within the first 24 months of ALS symptoms while patients were still in the early disease stage. Additionally, a pronounced difference was observed between groups in the dorsal half of the motor cortex - the half that corresponds to limb and trunk body regions of the homunculus. Dorsal M1 connectivity in the ALS group correlated with limb functioning, suggesting a brain-behavior relationship between these measures. It was found that the greater the disparity between right and left hand strength, the less functionally connected ROIs were in the dorsal motor cortex. This effect was not found in the healthy control group. Also, increased connectivity in the dorsal motor cortex was associated with better handwriting ability in those with ALS.

Results from the current study corroborate converging lines of evidence that intrinsic M1 functioning is altered in ALS. In a PET study, regional cerebral blood flow (rCBF) in M1 was reduced at rest in ALS patients compared to healthy participants. However, this reduction was not observed in lower motor neuron disease patients, implying upper motor neuron dysfunction in M1 (Kew et al., 1994). In an fMRI study, decreased blood oxygenated level dependent (BOLD) signal activity was found in contralateral M1 of ALS patients while performing a continuous unimanual buttonpressing task (Schoenfeld et al., 2005). In this particular study task difficulty effects were observed, and the authors suggested that decreased motor neurons in $\mathrm{M} 1$ contribute to sequential movement impairments. The present study provides new evidence for primary motor cortex changes between right and left cortices, importantly showing for the first time an interhemispheric disconnect (as shown by seed-based fcMRI) that is present even when participants were not performing a motor task.

Decreased interhemispheric connectivity in the primary motor cortex is consistent with recent findings from diffusion tensor imaging (DTI) work as well. For example, several studies have examined microstructural integrity within the corpus callosum, which include interhemispheric M1 projections. Reduced fractional anisotropy (FA), a measure of white matter microstructure, was found within the corpus callosum in ALS patients (Senda et al., 2009; Metwalli et al., 2010). These studies suggest that interhemispheric structural connectivity is compromised in those with ALS. In addition, Bartels et al. (2008) found that FA values in the corpus callosum correlated with behavioral measurements, specifically with the Contralateral Co-Movement Test, in patients with ALS. 
The only ALS resting-state fcMRI study to date demonstrated reduced functional connections between the premotor cortex and other sensorimotor regions during rest (Mohammadi et al., 2009). This may indicate that the sensorimotor network is becoming disconnected in ALS. Additionally, reduced functional connectivity was found in the prefrontal cortex, posterior/ventral-anterior areas of the cingulate cortex, and bilateral inferior parietal cortices, hence demonstrating decreased functional connectivity between core regions of the default mode network. Therefore, it is possible that regional increases in BOLD activity are related to neural-network breakdown in ALS.

There is a clinical need to establish biomarkers for those who will become diagnosed with ALS, and neuroimaging methodology may be well suited to identify these biomarkers (Agosta et al., 2010). This is important given the pronounced duration that occurs between symptom onset and diagnosis; for some people diagnosis is not established until years after symptoms present (Kraemer et al., 2010). This is especially true for those with limb onset, as symptoms often mimic other diseases (Leigh et al., 2003). Currently, the only reliable measurement used to diagnosis ALS is when a patient demonstrates progressive upper and lower motor neuron involvement (Turner et al., 2009). Results from the current study indicate that cortical neural changes are taking place in the early stages of ALS while patients are still highly functioning. Also, these changes are primarily occurring in the cortical regions that correspond to limb movement. Results from this study may be clinically relevant as a biomarker for limb-onset patients who have "suspected" or "probable" ALS. Identifying biomarkers specific to ALS will help increase the accuracy of diagnosis, decrease the duration between symptoms and diagnosis, and most importantly allow for initiation of early treatment (Turner et al., 2009; Ganesalingam and Bowser, 2010).

\section{LIMITATIONS AND FUTURE DIRECTIONS}

An important limitation in the current study is the lack of causal interpretation from our results. For example, this study emphasizes that interhemispheric M1 connectivity breakdown is more pronounced in the dorsal than ventral half between groups. Although the dorsal half corresponds to limb and trunk motor functioning, we cannot directly explain this relationship with the present findings. This is partially because it cannot be discerned whether our results reflect upper or lower motor neuron atrophy. Specifically, atrophy in lower motor neurons could give rise to M1 neural changes due to the decrease in afferent signals being received by M1. It could also be speculated that this area is more susceptible to the ALS disease process, or that it is an initial target of neurodegeneration. Another interpretation limitation is that we cannot differentiate whether these results are ALS-specific, or reflective of motor neuron disease processes in general. For example, neural atrophy has been identified in the primary motor cortex of patients with primary lateral sclerosis (Tartaglia et al., 2009), which may also give rise to functional connectivity changes. However, this hypoth-

\section{REFERENCES}

Agosta, F., Chio,A., Cosottini,M., DeStefano, N., Falini, A., Mascalchi, M., Rocca, M. A., Silani, V., Tedeschi, G., and Filippi, M. (2010). The present and future of neuroimaging in amyotrophic lateral sclerosis. Am. J. Radiol. 31, 1769-1777.
Bartels, C., Mertens, N., Hofer, S., Merboldt, K. D., Dietrich, J., Frahm, J., and Ehrenreich, H. (2008). Callosal dysfunction in amytrophic lateral sclerosis correlates with diffusion tensor imaging of the central motor system. Neuromuscul. Disord. 18, 398-407.

esis has yet to be tested and was beyond the scope of this current investigation. The specificity between motor neuron diseases will need to be further tested.

Context of using resting-state fcMRI must also be considered. It is assumed that temporally correlated signal fluctuations in brain regions imply functional connectivity. Therefore the interpretation that connections between right and left M1 are decreasing in ALS is based on this assumption. Furthermore, it is not known if this decrease in connectivity implies an axonal pathway impairment or gray matter loss within M1. Integrating other methods, such as DTI, would increase the effectiveness of addressing how the ALS disease process affects cortical networks.

Longitudinal methods are needed in order to clarify functional connectivity alterations in ALS. Results from the current study were from a single time point in the early stage of disease, yet it is suggested that these changes are progressive. Therefore we anticipate comparing results with data collected at subsequent sessions within the same participant group.

A fourth limitation is that the present results are only representative of ALS patients with limb-onset. There are both advantages and disadvantages to assessing neural changes in a homogenous patient group. This is advantageous in that disease processes specific to limb-onset ALS may be better described due to reduced variance by exclusion of a heterogeneous population. Also, a homogenous group increases our ability to control for any potential differences in disease progression between limb and bulbar onset patients. Through redefinition of inclusion criteria however, such as disease subtypes or individual behavioral symptoms, results may become more generalized to all disease processes. It could be potentially helpful for future studies to compare subtypes to disentangle pathological differences.

\section{CONCLUSIONS}

Results from the current study indicate that functional connections between the two primary motor cortices are decreased in ALS, and that this disconnect between hemispheres may be more specific to dorsal versus ventral M1. Dorsal M1 interhemispheric connectivity was related to metrics of upper limb functioning, specifically handwriting, and hand strength disparity. These brain-behavior correlations may have important clinical applications. Furthermore, neuroimaging methodology may be utilized for those with "possible" or "suspected" ALS by identifying reliable neural biomarkers.

\section{ACKNOWLEDGMENTS}

We greatly appreciate all participants who volunteered and gave their time for this study. We also thank Tom Nichols and Tim Johnson for statistical consultation, Scott Peltier for discussions and advice on functional connectivity methodology, and Pooja Modi for organizing the graph and tables. This work is supported by NIH/NINDS R01- NS052514 and Department of Radiology/ University of Michigan BRS Award.

Biswal, B. B., Van Kylen, J., and Hyde, J. S. (1997). Simultaneous assessment of flow and BOLD signals in resting-state functional connectivity maps. NMR Biomed. 10, 165-170.

Biswal, B. B., Yetkin, F. Z., Haughton, V. M., and Hyde, J. S. (1995). Functional connectivity in the motor cortex of resting human brain using echoplanar MRI. Magn. Reson. Med. 34, 537-541.

Brownell, B., Oppenheimer, D. R., and Hughes, J. T. (1970). The central nervous system in motor neurone disease. 
J. Neurol. Neurosurg. Psychiatr. 33, 338-357.

Bruijn, L. I., Miller, T. M., and Cleveland, D. W. (2004). Unraveling the mechanisms involved in motor neuron degeneration in ALS. Annu. Rev. Neurosci. 27, 723-749.

Cao, Q., Zang, Y., Sun, L., Sui, M., Long, X., Zou, Q., and Wang, Y. (2006). Abnormal neural activity in children with attention deficit hyperactivity disorder: a resting-state functional magnetic resonance imaging study. Neuroreport 17, 1033-1036.

Cedarbaum, J.M., and Stambler, N. (1997). Performance of the amyotrophic lateral sclerosis functional rating scale (ALSFRS) in multicenter clinical trials. J. Neurol. Sci. 152(Suppl. 1), s1-s9.

Chakravarti, I. M., Laha, R. G., and Roy, J. (1967). Handbook of Methods of Applied Statistics (I). New York: John Wiley and Sons.

Cherkassky, V. L., Kana, R. K., Keller, T. A., and Just, M. A. (2006). Functional connectivity in a baseline resting-state network in autism. Neuroreport 17, 1687-1690.

Craddock, R. C., James, G. A., Holtzheimer, P. E., Hu, X. P., and Mayberg, H. S. (2010). "A whole brain fMRI atlas generated via spatially constrained spectral clustering: Part I, Method," in Proceedings of the 16th Annual Meeting of the Organization for Human Brain Mapping, Barcelona, Spain, 89.

Damoiseaux, J. S., Rombouts, S. A. R. B., Barkhof, F., Scheltens, P., Stam, C. J., Smith, S. M., and Beckmann, C. F. (2006). Consistent resting-state networks across healthy subjects. Proc. Natl. Acad. Sci. U.S.A. 103, 13848-13853.

del Aguila, M. A., Longstreth, W. T. J., McGuire, V., Koepsell, T. D., and van Belle, G. (2003). Prognosis in amyotrophic lateral sclerosis: a populationbased study. Neurology 60, 813-819.

Fox, M. D., Snyder, A. Z., Vincent, J. L., Corbetta, M., Van Essen, D. C., and Raichle, M. E. (2005). The human brain is intrinsically organized into dynamic, anticorrelated functional networks. Proc. Natl. Acad. Sci. U.S.A. 102, 9673-9678.

Fox, M. D., Zhang, D., Snyder, A. Z., and Raichle, M.E. (2009). The global signal and observed anticorrelated resting state brain networks. J. Neurophysiol. 101, 3270-3283.

Ganesalingam, J., and Bowser, R. (2010). The application of biomarkers in clinical trials for motor neuron disease. Biomark. Med. 4, 281-297.

Grady, C. L., Furey, M. L., Pietrini, P., Horwitz, B., and Rapoport, S. I. (2001). Altered brain functional connectivity and impaired short-term memory in Alzheimer's disease. Brain 124, 739-756.
Greicius, M. D., Flores, B. H., Menon, V., Glover, G. H., Solvason, H. B., Kenna, H., Reiss, A. L., and Schatzberg, A. F. (2007). Resting-state functional connectivity in major depression: abnormally increased contributions from subgenual cingulate cortex and thalamus. Biol. Psychiatry 62, 429-437.

He, B. J., Snyder,A.Z., Vincent, J.L., Epstein, A., Shulman, G. L., and Corbetta, M. (2007). Breakdown of functional connectivity in frontoparietal networks underlies behavioral deficits in spatial neglect. Neuron 53, 905-918.

$\mathrm{Hu}, \mathrm{X} ., \mathrm{Le}, \mathrm{T} . \mathrm{H} ., \mathrm{Parrish}, \mathrm{T}$., and Erhard, P. (1995). Retrospective estimation and correction of physiological fluctuations in functional MRI. Magn. Reson. Med. 34, 201-212.

Kew, J. J. M., Brooks, D. J., Passingham, R. E., Rothwell, J. C., Frackowiak, R. S. J., and Leigh, P. N. (1994). Cortical function in progressive lower motor neuron disorders and amyotrophic lateral sclerosis: a comparative PET study. Neurology 44, 1101-1110.

Koshino, H., Carpenter, P. A., Minshew, N. J., Cherkassky, V. L., Keller, T. A., and Just, M.A. (2005). Functional connectivity in an fMRI working memory task in high-functioning autism. Neuroimage 24, 810-821.

Kraemer, M., Buerger, M., and Berlit, P. (2010). Diagnostic problems and delay of diagnosis in amyotrophic lateral sclerosis. Clin. Neurol. Neurosurg. 112, 103-105.

Lawyer, T., and Netsky, M. G. (1953). Amyotrophic lateral sclerosis: a clinicoanatomic study of fifty-three cases. AMA Arch. Neurol. Psychiatry 69, 171-192.

Leigh, P. N., Abrahams, S., Al-Chalabi, A., Ampong, M. A., Goldstein, L. H., Johnson, J., Lyall, R., Moxham, J., Mustfa, N., Rio,A.,Shaw, C., and Willey, E. (2003). The management of motor neurone disease. J. Neurol. Neurosurg. Psychiatr. 74(Suppl. 4), iv32-iv47.

Lowe, M. J., Phillips, M. D., Lurito, J. T., Mattson, D., Dzemidzic, M., and Mathews, V.P. (2002). Multiple sclerosis: low-frequency temporal blood oxygen level-dependent fluctuations indicate reduced functional connectivity-initial results. Radiology 224, 184-192.

Lund, T. E. (2001). fcMRI--mapping functional connectivity or correlating cardiac-induced noise? Magn. Reson. Med. 46, 628-629.

Metwalli, N. S., Benatar, M., Nair, G., Usher, S., Hu, X., and Carew, J. D. (2010). Utility of axial and radial diffusivity from diffusion tensor MRI as markers of neurodegeneration in amyotrophic lateral sclerosis. Brain Res. 1348, 156-164.

Mohammadi, B., Kollewe, K., Samii, A., Krampfl, K., Dengler, R., and Munte,
T. F. (2009). Changes of resting state brain networks in amyotrophic lateral sclerosis. Exp. Neurol. 217, 147-153.

Monk, C. S., Peltier, S. J., Wiggins, J. L., Weng, S. J., Carrasco, M., Risi, S., and Lord, C. (2009). Abnormalities of intrinsic functional connectivity in autism spectrum disorders. Neuroimage 47, 764-772.

Noll, D. C., Meyer, C. H., Pauly, J. M., Nishimura, D. G., and Macovski, A. (1991). A homogeneity correction method for magnetic resonance imaging with time-varying gradients. IEEE Trans. Med. Imaging 10, 629-637.

Oldfield, R. C. (1971). The assessment and analysis of handedness; the Edinburgh inventory. Neuropsychology 9, 97-113.

Peltier, S. J., Polk, T. A., and Noll, D. C. (2003). Detecting low-frequency functional connectivity in fMRI using a self-organizing map (SOM) algorithm. Hum. Brain Mapp. 20, 220-226.

Penfield, W., and Boldrey, E. (1937). Somatic motor and sensory representation in the cerebral cortex of man as studied by electrical stimulation. Brain 60, 389-443.

Pfeuffer, J., Van De Moortele, P. F., Ugurbil, K., Hu, X., and Glover, G. H. (2002). Correction of physiologically induced global off-resonance effects in dynamic echo-planar and spiral functional imaging. Magn. Reson. Med. 47, 344-353.

Schoenfeld, M.A., Tempelmann, C., Gaul, C., Kühnel, G. R., Düzel,E.,Hopf,J.-M., Feistner, H., Zierz, S., Heinze, H. J., and Vielhaber, S. (2005). Functional motor compensation in amyotrophic lateral sclerosis. J. Neurol. 252, 944-952.

Seeley, W. W., Crawford, R. K., Zhou, J., Miller, B. L., and Greicius, M. D (2009). Neurodegenerative diseases target large-scale human brain networks. Neuron 62, 42-52.

Senda, J., Ito, M., Watanabe, H., Atsuta, N., Kawai, Y., Katsuno, M., Tanaka, F., Naganawa, S., Fukatsu, H., and Sobue, G. (2009). Correlation between pyramidal tract degeneration and widespread white matter involvement in amyotrophic lateral sclerosis: a study with tractography and diffusiontensor imaging. Amyotroph. Lateral Scler. 10, 288-294.

Smith, M. C. (1960). Nerve fiber degeneration in the brain in amyotrophic lateral sclerosis. J. Neurol. Neurosurg. Psychiatr. 23, 269-282.

Tartaglia, M. C., Laluze, V., Rowe, A., Findlater, K., Lee, D. H., Kennedy., K. Kramer, J. H., and Strong, M. J. (2009). Brain atrophy in primary lateral sclerosis. Neurology 72, 1236-1241.

Tian, L., Jiang, T., Wang, Y., Zang, Y., He, Y., Liang, M., Sui, M., Cao, Q., Hu, S. Peng, M., and Zhuo, Y. (2006). Altered resting-state functional connectivity patterns of anterior cingulate cortex in adolescents with attention deficit hyperactivity disorder. Neurosci. Lett. 400, 39-43.

Turner, M. R., Kiernan, M. C., Leigh, P. N., and Talbot, K. (2009). Biomarkers in amyotrophic lateral sclerosis. Lancet Neurol. 8, 94-109.

van der Graaff, M. M., de Jong, J. M. B.

V., Baas, F., and de Visser, M. (2009). Upper motor neuron and extra-motor neuron involvement in amyotrophic lateral sclerosis: a clinical and brain imaging review. Neuromuscul. Disord. 19, 53-58.

Waites, A. B., Briellmann, R. S., Saling, M. M., Abbott, D. F., and Jackson, G. D. (2006). Functional connectivity networks are disrupted in left temporal lobe epilepsy. Ann. Neurol. 59, 335-343.

Wang, K., Jiang, T., Liang, M., Wang, L. Tian, L., Zhang, X., Li, K., and Liu, Z. (2006a). Discriminative analysis of early Alzheimer's disease based on two intrinsically anti-correlated networks with resting-state fMRI. Med. Image Comput. Comput. Assist. Interv. 9, 340-347.

Wang, L., Zang, Y., He, Y., Liang, M., Zhang, X., Tian, L., Wu, T., Jiang, T. and Li, K. (2006b). Changes in hippocampal connectivity in the early stages of Alzheimer's disease: evidence from resting state fMRI. Neuroimage 31, 496-504.

Welsh, R. C., Chen, A. C., and Taylor, S. F. (2008). Low-frequency BOLD fluctuations demonstrate altered thalamocortical connectivity in schizophrenia. Schizophr. Bull. 36, 713-722.

Woolley-Levine, S. (2006). ALS Cognitive Behavioral Screen. San Francisco: California Pacific Medical Center.

Conflict of Interest Statement: The authors declare that the research was conducted in the absence of any commercial or financial relationships that could be construed as a potential conflict of interest.

Received: 01 July 2010; accepted: 06 December 2010; published online: 31 December 2010.

Citation: Jelsone-Swain LM, Fling BW, Seidler RD, Hovatter R, Gruis Kand Welsh RC (2010) Reduced interhemispheric functional connectivity in the motor cortex during rest in limb-onset amyotrophic lateral sclerosis. Front. Syst. Neurosci. 4:158. doi 10.3389/fnsys.2010.00158

Copyright $\odot 2010$ Jelsone-Swain, Fling, Seidler, Hovatter, Gruis and Welsh. This is an open-access article subject to an exclusive license agreement between the authors and the Frontiers Research Foundation, which permits unrestricted use, distribution, and reproduction in any medium, provided the original authors and source are credited. 